\title{
A Model Based Dual Active Contour
}

\author{
S. R. Gunn and M. S. Nixon \\ Vision Speech and Signal Processing Group \\ Department of Electronics and Computer Science \\ University of Southampton, SO17 1BJ
}

\begin{abstract}
Active contours are now established as a technique for extracting salient contours from an image. Unfortunately the original technique suffers from many problems. A novel model-based dual active contour, a method of integrating global shape information with two active contours, has been developed to overcome the primary problems; sensitivity to initialisation and undesirable attractions by insignificant localised or regionalised features. The model guides the technique to avoid insignificant minima and is relinquished when the energy minimum is sufficiently compatible. The technique then finally operates as a pair of conventional active contours, ensuring that only image information is extracted, consistent with the original technique.
\end{abstract}

\section{Introduction}

An active contour (also known as a snake by virtue of the nature of its evolution) is an energy minimising spline [6] that is constrained by its own internal forces of continuity and curvature, whilst external forces drive it towards desired image features. The external forces are provided by the image and where appropriate, information from a higher-level process may be included. The image functional is constructed so that local minima correspond to desired features. The snake's energy depends on its shape and location within the image. The problem is formulated as an energy minimisation problem. The minimisation process evolves an initial contour towards an appropriate feature. Typically there are many local minima in the energy function. In this situation the contour may become trapped in strong neighbouring edges resulting in a false boundary. The technique may be improved by making it more selective. Prior knowledge may be included in the process in order to achieve this. A model-free interpretation is limited because the problem is underconstrained. Various methods have been proposed to avoid insignificant false edges; a spatially smoothed edge representation [6], successive 
lengthening of an active contour [1], the inclusion of region-based information [4, 7], and an internal pressure force [2] have been introduced. However, even with the application of these techniques the contour may still become trapped by false edges.

The inclusion of a global shape model biases the contour towards a target shape, giving the contour some selectivity over image features. One method [8] used a probabilistic shape model to integrate global shape information with the contour; this reduces speed, but demonstrates the increased robustness provided by the inclusion of shape information. The model-based dual snake is directly applicable in image space as opposed to parameter space, and is developed as an extension to the idea of a dual snake.

\section{The Snake Model}

A contour is described parametrically by $\mathbf{v}(s)=(x(s), y(s))$ where $x(s), y(s)$ are $x, y$ co-ordinates along the contour and $s \in[0,1]$ is arc length. The snake model defines the energy of a contour $\mathrm{v}(s)$, the snake energy $E_{\text {snake, }}$, to be,

$$
E_{\text {snake }}(\mathbf{v}(s))=\int_{s=0}^{1} E_{\text {int }}(\mathbf{v}(s))+E_{\text {image }}(\mathbf{v}(s))+E_{\text {model }}(\mathbf{v}(s)) d s
$$

where $E_{\text {int }}$ is the internal energy of the contour, imposing continuity and curvature constraints, $E_{\text {image }}$ is the image energy constructed to attract the snake to desired feature points in the image; the functional, $E_{\text {image }}=-\mid$ $\nabla I(x, y) \mid$ attracts the snakes to edges in the image, and $E_{\text {model }}$ is the model energy which allows geometric constraints to be applied during the minimisation process. An initial contour evolves by minimising eqn. 1 using a gradient descent technique.

\section{A Dual Active Contour}

In the dual contour approach, an interior contour lies within the region of the desired feature and an exterior contour outside it. The two contours are coupled using springs which cause the two contours to be attracted to each other as well as to suitable image features, Fig. 1. The purpose of two contours is to integrate the information from a contour expanding within the feature to a contour contracting from without the feature. The dual snake resolves the sensitivity to normal force associated with the 
method of [2,3], whilst reducing sensitivity to initialisation. It also provides superior performance in the case of non-convex shapes by incorporating the information from two snakes approaching the desired boundary from both sides. Initialisation involves placing an initial interior contour within the feature and an exterior contour outside the feature.

We define the interior and exterior contours as inner and outer respectively. The spring forces are included by adding a constraint energy term, $E_{\text {model }}$, where the spring force is controlled by $\lambda$,

$$
E_{\text {model }}(\mathbf{v}(s))=\lambda \frac{1}{2}(\mathbf{v}(s)-\operatorname{mean}(s))^{2}
$$

and mean(s) is the mean contour of the interior and exterior contours.

$$
\operatorname{mean}(s)=\frac{1}{2}(\operatorname{inner}(s)+\operatorname{outer}(s))
$$

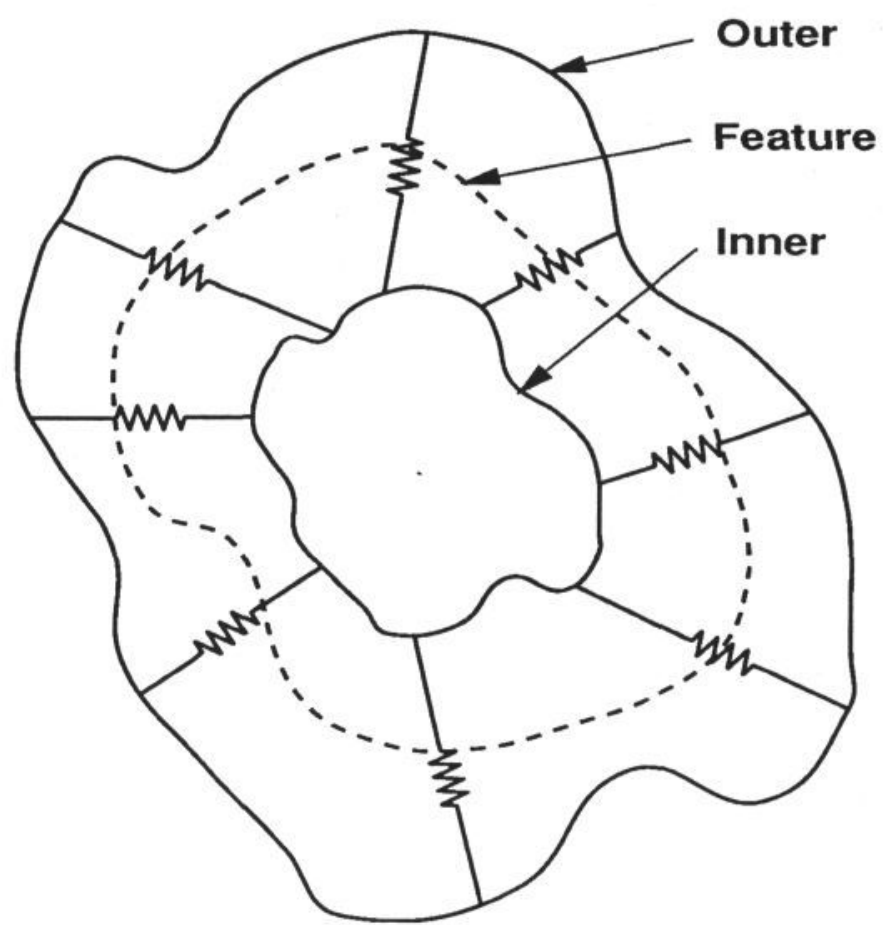

Figure 1. Dual snake configuration. 


\section{A Model-based Dual Active Contour}

The design of the snake has the advantage that high-level information, such as geometric shape may be included in the extraction process to improve performance in the presence of imperfect image data. In order to achieve the maximum benefit from the inclusion of a model in the feature extraction process it should be incorporated as early as possible. The original snake incorporates non-specific information such as smoothness or low overall curvature. This is optimal when no further information is available, but for most applications there is more specific information available about the desired shape. It is imperative to include as much specific knowledge as possible in these cases to enhance the performance of the technique. A geometric shape model is an appropriate method of including this information.

Many shapes have sharp edges that are not suited to the snake's natural global characteristic, low overall curvature. Inclusion of a model allows the snake to be biased towards an estimated model and makes available shape information for modifying the snake's internal constraints, e.g. by allowing corners to form. The model increases robustness in the presence of occlusion, poor contrast or noise. In practice, the dual-snake enables a superior estimate of the desired feature's location to be obtained, with respect to the original snake.

The information in the interior and exterior contours determines an annulus within which the feature is predicted to lic. Using the inherent shape information in the two contours the model is fitted to a mean contour, the average of the interior and exterior contours. The interior and exterior contours are connected to the model using springs, Fig 2. An ellipse with a fixed eccentricity is used as a model here.

The fitting process must scale, translatc and rotate a rigid shape model to fit a predicted position. Rotation is the critical affine transformation. The technique of least squares is used to find a best-fit model. Least squares is an appropriate technique here since the fitting is to a mean contour derived from highly correlated data. To align the two contours $\mathbf{v}(0)$ is chosen as the point with minimum argument, with respect to the centroid of the contour.

The mean contour is computed from the interior and extcrior contours, inner and outer, as

$$
\operatorname{mean}(s)=\frac{1}{2}(\operatorname{inner}(s)+\operatorname{outer}(s))
$$




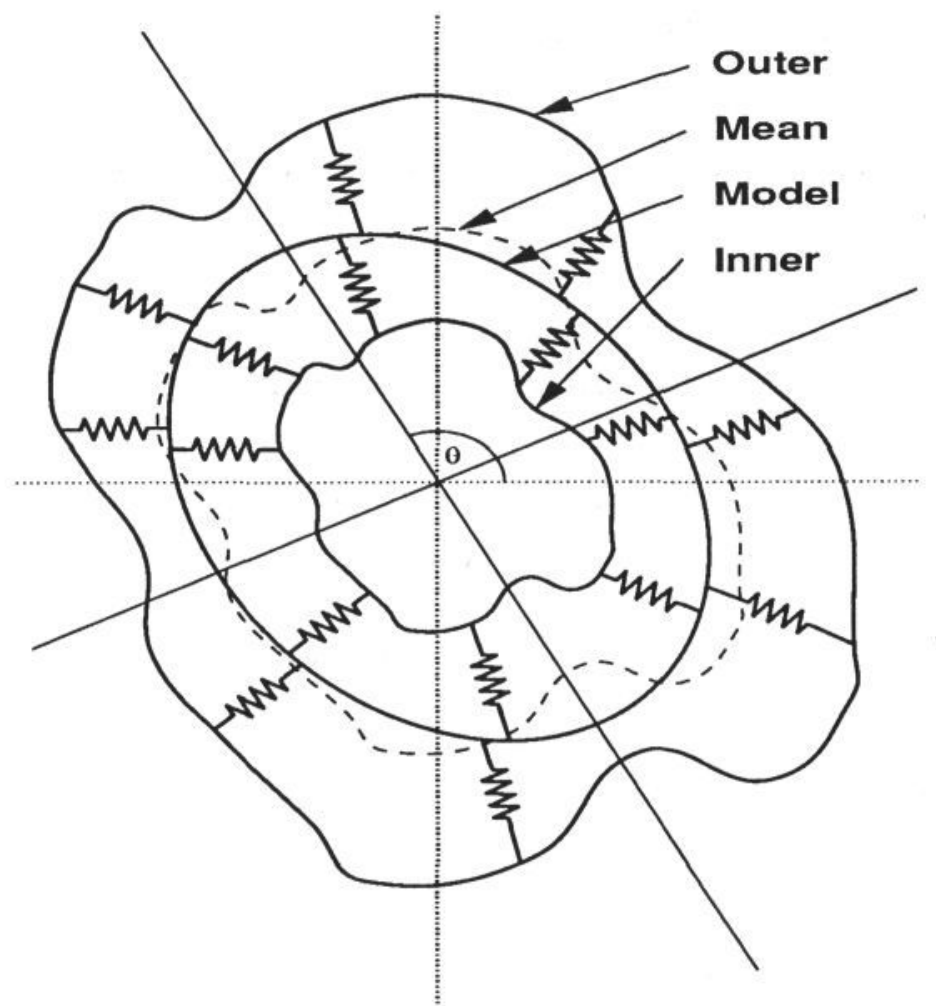

Figure 2. Model based dual active contour configuration.

By defining $\mu_{p, q}$, the $(\mathrm{p}, \mathrm{q})$ order central boundary based moment of a contour

$$
\mu_{p, q}(\mathbf{v}(s))=\int_{s=0}^{1}(x(s)-\bar{x})^{p}(y(s)-\bar{y})^{q} d s
$$

where $(\bar{x}, \bar{y})$ is the centroid of the contour.

$$
\bar{x}=\int_{s=0}^{1} x(s) d s \quad \bar{y}=\int_{s=0}^{1} y(s) d s
$$

The orientation of a contour is defined as the inclination of the axis of minimum moment of inertia. The moment of inertia, $M$, about an axis with inclination $\theta$ to the $\mathrm{x}$ axis is 


$$
M(\theta)=\int_{s=0}^{1}[(y(s)-\bar{y}) \cos \theta-(x(s)-\bar{x}) \sin \theta]^{2} d s
$$

By minimising eqn. 7 with respect to the inclination, $\theta$, the angle of minimum moment of inertia, $\theta_{\min }$, is

$$
\theta_{\min }=\frac{1}{2} \tan ^{-1}\left(\frac{2 \mu_{1,1}(\text { mean })}{\mu_{2,0}(\text { mean })-\mu_{0,2}(\text { mean })}\right)
$$

Then the least squares fit of an ellipse with eccentricity $e$, to the mean contour, gives the minor and major axes, $a$ and $b$, respectively as

$$
\begin{aligned}
& b=\sqrt{\left(e^{2} c_{\theta}^{2}+1\right) \mu_{2,0}(\text { mean })+2 e^{2} c_{\theta} s_{\theta} \mu_{1,1}(\text { mean })+\left(e^{2} s_{\theta}^{2}+1\right) \mu_{0,2}(\text { mean })}, \\
& a=\sqrt{\left(1-e^{2}\right)} b,
\end{aligned}
$$

where $c_{\theta}=\cos \left(\theta_{\min }\right), s_{\theta}=\sin \left(\theta_{\min }\right)$.

A model contour, model, is defined as a representation of the parametric ellipse.

$$
\operatorname{model}(s)=\left(\begin{array}{l}
a \cdot c_{\theta} \cdot \cos \left(2 \pi s-\theta_{\text {min }}\right)-b \cdot s_{\theta} \cdot \sin \left(2 \pi s-\theta_{\text {min }}\right)+\bar{x} \\
a \cdot s_{\theta} \cdot \cos \left(2 \pi s-\theta_{\text {min }}\right)+b \cdot c_{\theta} \cdot \sin \left(2 \pi s-\theta_{\text {min }}\right)+\bar{y}
\end{array}\right)
$$

The model energy is of the form of eqn. 2 but with the mean contour replaced by the model and $\lambda$ by $\lambda(s)$ since $\lambda$ can vary with position.

No readjustment in the alignment of the contours is necessary since alignment is retained by the springs connecting the model. The implementation uses a discrete polygonal contour. The inclusion of the model prevents the points bunching on parts of the contour with high curvature, which is a problem with the original technique. This is achieved by ensuring the points on the model are approximately evenly spaced. When no reduction in energy is perceived then the model is relinquished $\left(E_{\text {model }}=0\right)$ and the technique continues to operate for both contours as a basic snake formulation. 


\section{Results}

The images used were $256 \times 256$ pixels of 8 bits. Pre-processing involved intensity normalisation. The simplest image functional was implemented consisting solely of a magnitude gradient edge functional. The absence of an objective performance measure for snakes has premeditated the study of techniques for performance evaluation. For a true performance measure we need to take into account initialisation, the shape of the image functional, the final contour, the desired contour and possibly the number of iterations. Therefore only the difference in the expected contour from the final contour is considered. The dependence on initialisation and image is minimised by using a single initialisation and a single image, where both the image and initialisation are carefully chosen to highlight the difficulties with the original technique.

The chosen cup image contains clear edges of the cup along with unwanted ones from the background and the cup's inscription. Fig. 3. shows the results. The image functional used is shown in Fig 3(a), along with the initialisation for the original technique in Fig 3(b) and the dual and model based technique in Fig 3(d). The initial contours for the dual techniques should lie entirely within and without the feature for good performance. The final contour extracted by the basic technique, Fig. 3(c), shows how the contour can focus on insignificant edges in the inscription. The dual active contour, Fig 3(e), and the model based version, Fig 3(f), demonstrate the increased robustness (for extracting the cup rim) provided by the ability to pass over these insignificant features. A single expanding contour initialised as the inner contour in Fig. 3(d) can extract the rim with a careful choice of parameters, however the snake becomes more rigid due to the inflationary force counteracting the elasticity of the snake. Table 1 confirms the results in terms of an area difference measure $A(\alpha, \beta$ ), eqn. 11 , between the final extracted contour $\beta(\theta)$, and desired contour $\alpha$ $(\theta)$. This measures error in the extracted contour and is smallest for the model based dual active contour.

$$
A(\alpha, \beta)=\frac{\int_{\theta=0}^{2 \pi}\left|\alpha(\theta)^{2}-\beta(\theta)^{2}\right| d \theta}{\int_{\theta=0}^{2 \pi} \alpha(\theta)^{2} d \theta}
$$




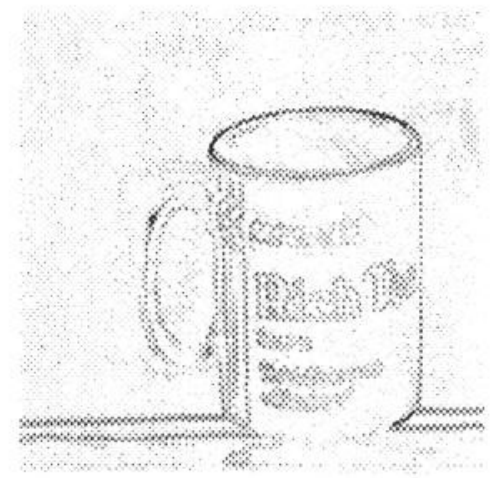

(a) Image edge functional.

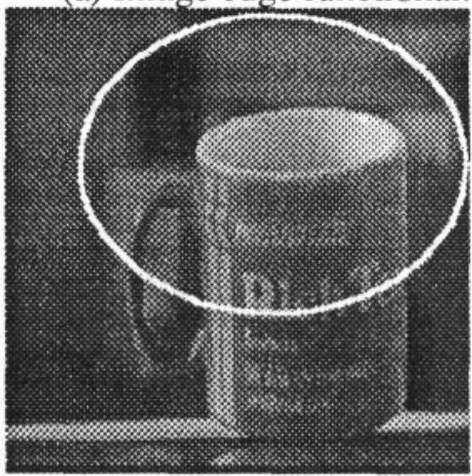

(b) Initial contour.

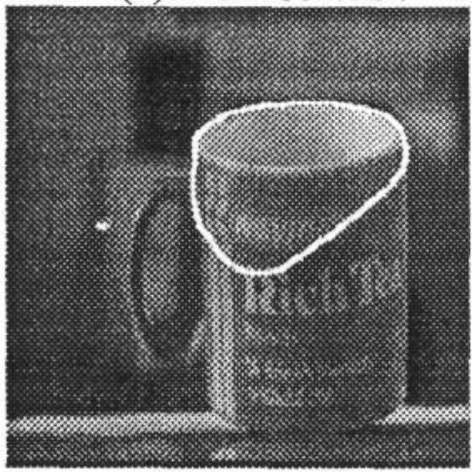

(c) Final contour.

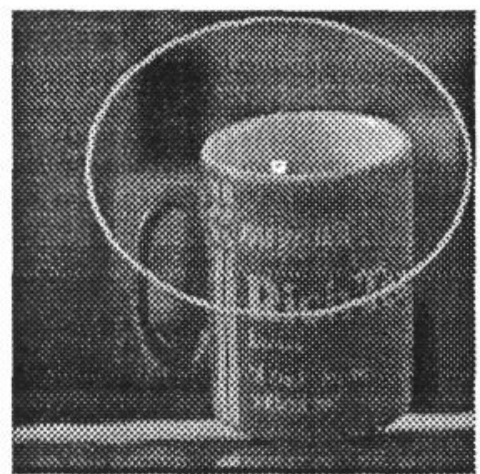

(d) Initial contours.

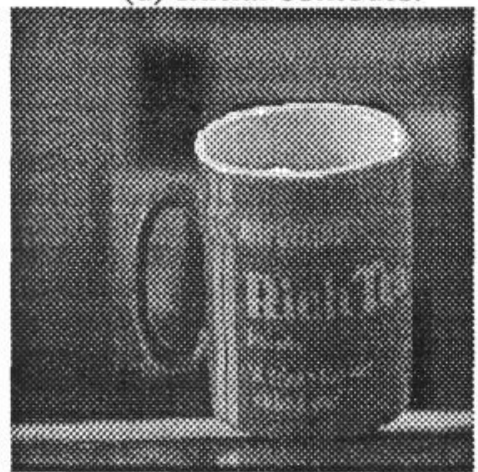

(e) Final dual contours.

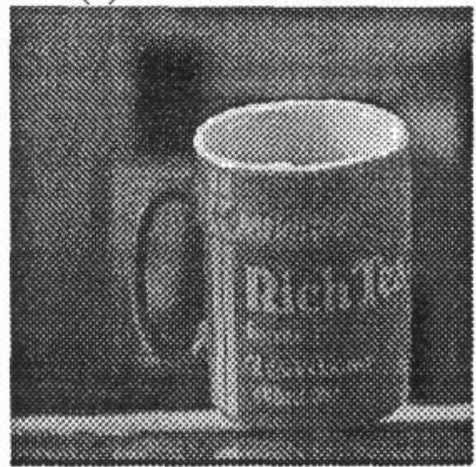

(f) Final model based contours.

Figure 3. Results 


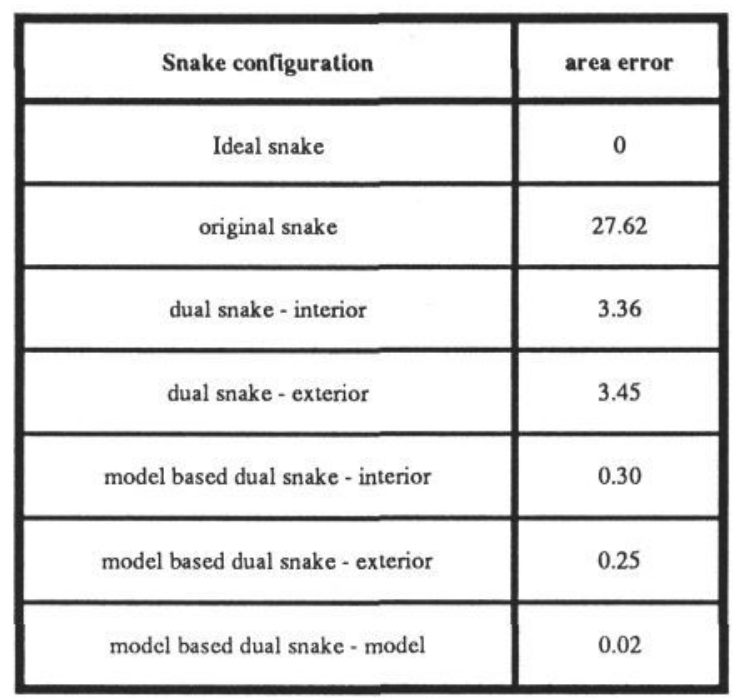

Table 1. Area difference measure results.

\section{Extensions}

The technique can clearly be generalised to include non-parametric shapes. Also, the spring force can be dependent on the model and reduced when model data is only known with reduced confidence. One suitable application is in head boundary extraction. This has already been tackled with deformable templates, however the high order description associated with active contours can provide better information for recognition purposes. A hybrid head model [5] used a parametric curve for the lower part of the face whereas the upper part, where no clear model exists, was extracted by the dual contour approach.

If the model is included too early in the extraction process the estimated orientation may be in error and inhibit the contours from finding the desired feature. This warrants future research into a process to automatically choose when the model can be incorporated. A suitable criterion should include the shape of the interior and exterior contours. At present the model is applied immediately.

\section{Conclusions}

The main problems with the original snake model have been highlighted; sensitivity to initialisation, undesirable attractions by insignificant 
localised or regionalised features, no relationship between their state of equilibrium and the contour to be extracted.

A novel model-based dual snake integrates global shape information with an active contour to guide the contour into an appropriate minimum. It has been shown how the potential to integrate knowledge with the extraction process may be exploited to improve the selectivity of the contour and hence increase its robustness. However further investigation is warranted in improving the model fitting process by consulting local image information as well as the contour shapes to further improve robustness. Particular emphasis should be placed on when to start to fit the model using the inherent information in the contours.

\section{References}

[1] M. O. Berger. Snake growing. Lecture Notes in Computer Science, 427:387-396, 1990.

[2] L. D. Cohen and I. Cohen. Finite-Element Methods for Active Contour Models and Balloons for 2D and 3D Images. IEEE Transactions on Pattern Analysis and Machine Intelligence, 15(11):1131-1147, 1993.

[3] L. D. Cohen. NOTE: On Active Contour Models and Balloons. CVGIP: Image Understanding, 53(2):211-218, 1991.

[4] M. Etoh, Y. Shirai and M. Asada. Contour Extraction by Mixture Density Description Obtained from Region Clustering. ECCV 92 2nd Euro. Conference on Computer Vision, Santa Margherita Ligure, Italy, 24-32, 1992.

[5] S. R. Gunn and M. S. Nixon. A Dual Active Contour for Hcad Boundary extraction. IEE Colloquium 1994/100, 6/1-6/4.

[6] M. Kass, A. Witkin and D. Terzopoulos. Snakes: Active Contour Models. International Journal of Computer Vision, 1:321-331, 1988.

[7] N. Rougon and F. Pretcux. Deformable markers: Mathematical Morphology for active contour models control. SPIE Image Algebra and Morphological Image Processing II, 1568:78-89, 1991.

[8] L. H. Staib and J. S. Duncan. Boundary Finding with Parametrically Deformable Models. IEEE Transactions on Pattern Analysis and Machine Intelligence, 14(11):1061-1075, 1992. 